\title{
Growth of ZnO Nanorods: A TEM Study
}

\author{
R. Divakar, Joysurya Basu, and C. Barry Carter
}

Chemical Engineering and Materials Science, University of Minnesota, Minneapolis, MN 55455.

In the present age of nanostructured semiconductors, $\mathrm{ZnO}$ has an important role because of its wide band gap of $\sim 3.37 \mathrm{eV}$, piezoelectricity and facile synthesis as well formed, crystallographically oriented single nanocrystals in pure or alloyed (doped) conditions. Some existing and potential applications include sensors, piezoelectric transducers and optical applications [1-4]. Full realization of potential applications requires a thorough understanding of growth mechanisms as well as techniques for integrating the nanostructures with other materials. This would enable synthesis of nanorods of the required geometry for nanostructured device applications. The present paper discusses crystal growth mechanisms in relation to substrates and also the interface structures that arise. $\mathrm{ZnO}$ nanostructures have been characterized by transmission electron microscopy techniques using a Tecnai G2 F30 microscope operated at $300 \mathrm{kV}$ and a Tecnai T12 operating at $120 \mathrm{kV}$.

$\mathrm{ZnO}$ nanostructures have been synthesized via a hydrothermal synthesis route on substrates exposed to the vapor. Single-crystalline $\mathrm{ZnO}$ nanorods shaped as hexagonal prisms, several tens of nanometers wide and 2-3 $\mu \mathrm{m}$ long were formed (Figure 1). The nanorods were seen to grow even in the absence of seed crystals. Crystallographic and chemical identity corresponding to hexagonal $\mathrm{ZnO}$ have been confirmed using electron diffraction and energy dispersive spectroscopy. Several morphological features are of interest. The nanorods are seen to taper at both ends starting from a central point that is almost exactly midway between the two ends. Diffraction and HRTEM evidence point to a growth direction that is normal to (0001) planes (Figure 2). There is evidence for some "proto-nanorods" oriented with the basal plane parallel to the substrate. The bounding planes of these hexagonal nanocrystals are of the $\{10 \overline{1} 0\}$ type. Further, morphological features related to growth of nanorods on various substrate surfaces will also be shown.

While the chemistry of precipitation of $\mathrm{ZnO}$ during hydrothermal synthesis is well known, the processes responsible for the formation through the vapor phase and the nanorod morphology are less understood. Growth is seen to occur on all surfaces exposed to the vapor. In literature, nanowire growth is suggested to occur by the oriented coupling of crystallites [5]. The growth planes and the bounding planes of the nanorods determined in the present study suggest an influence from the surface energy as well as kinetic factors in the growth of the nanostructures formed. The present study reports on these aspects of nanorod growth in the absence of seed crystals and on crystallographically distinct substrates. [6].

References

[1] J. Grabowska et al., J. Mater. Sci.: Materials in Electronics, 16 (2005) 397.

[2] J. Xu et al., J. Mater. Sci., 40 (2005) 2919

[3] Z. L. Wang, J. Mater. Chem., 15 (2005) 1021.

[4] J. Basu et al., Microsc. Microanal., 12 (Suppl. 2) (2006).

[5] M. Andres Verges et al., J. Chem. Soc. Farady Trans., 86 (1990) 959. 
[6] This research is supported by the University of Minnesota through the MRSEC Program of the National Science Foundation under Award \# DMR-0212302. We acknowledge discussions with Dr. X. Wang and Prof. H. Jacobs.

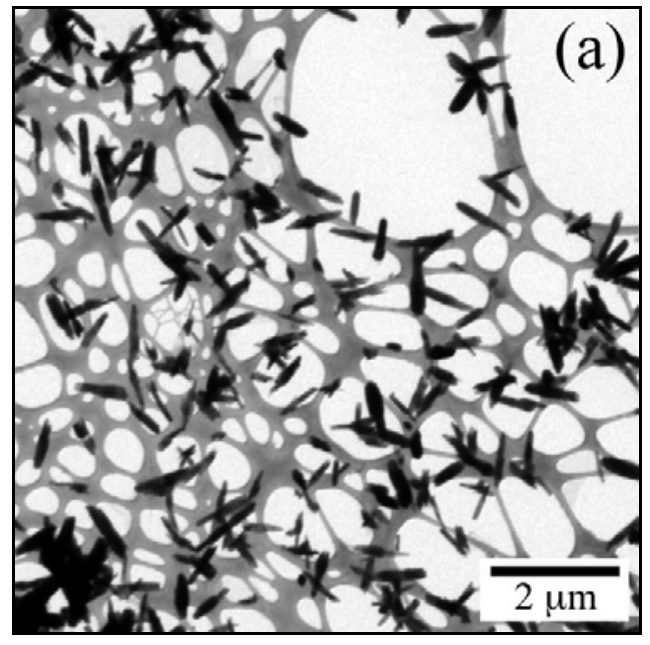

FIG. 1. (a) $\mathrm{ZnO}$ nanorods grown on amorphous carbon film. (b) Bright-field image showing well formed nanorods with tapered ends. (c) Energy dispersive spectrum from a single nanorod confirming the $\mathrm{ZnO}$ phase.

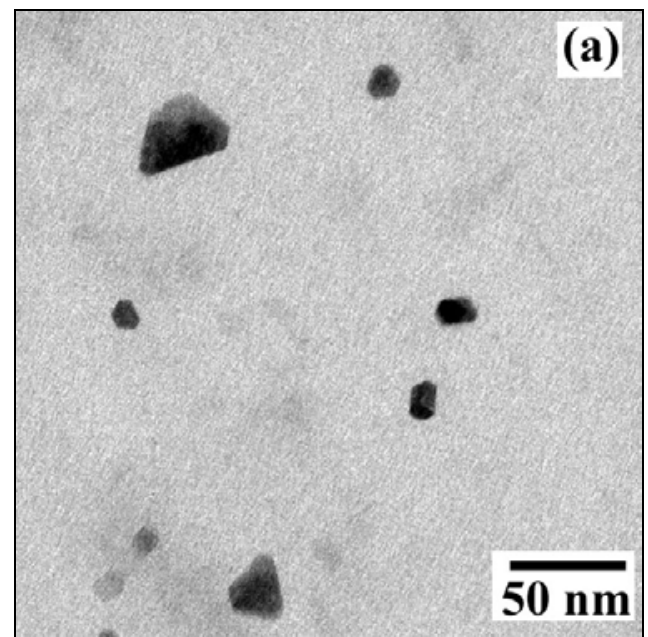

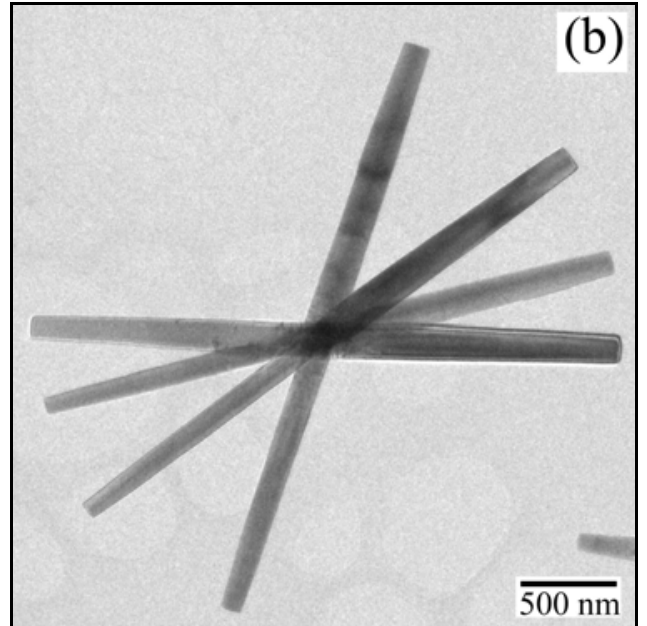
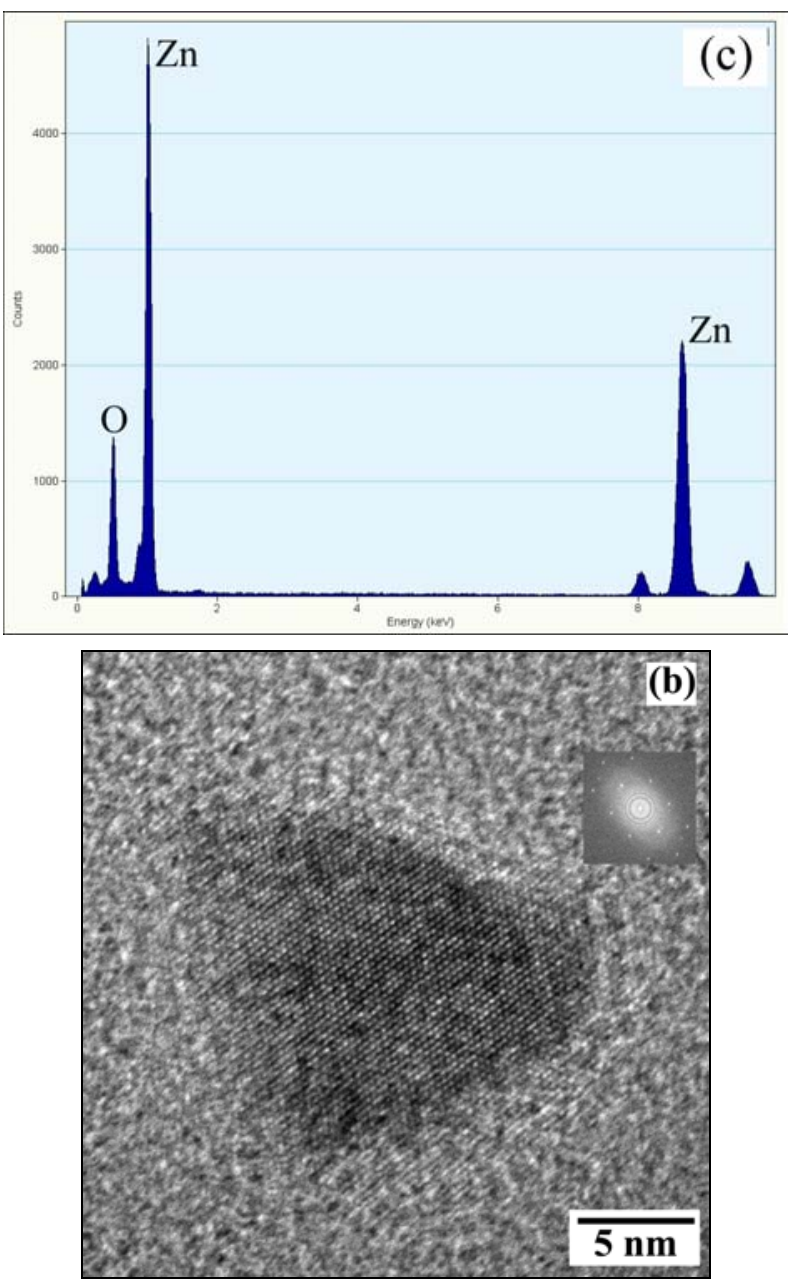

FIG. 2. (a) $\mathrm{ZnO}$ formed as facetted nanoparticles, (b) HRTEM image of a facetted nanoparticle. Inset shows the power spectrum. The facet planes correspond to $\{10 \overline{1} 0\}$. 